\title{
Research Paper: Cardiovascular Effect of Cuneiform Nucleus During Hemorrhagic Hypotension
}

\author{
Reza Mohebbati' $\odot$, Mahmoud Hosseini ${ }^{2} \odot$, Majid Khazaei $^{1} \odot$, Mohammad Naser Shafei ${ }^{3 *} \odot$ \\ 1. Department of Physiology, Faculty of Medicine, Mashhad University of Medical Sciences, Mashhad, Iran. \\ 2. Division of Neurocognitive Sciences, Psychiatry and Behavioral Sciences Research Center, Mashhad University of Medical Sciences, Mashhad, Iran. \\ 3. Neurogenic Inflammation Research Centre, Mashhad University of Medical Sciences, Mashhad, Iran.
}

\begin{tabular}{|c|c|}
\hline Use your device to scan & \\
\hline and reat the article online & $\begin{array}{l}\text { ditation: Mohebbati, R., Hosseini, M., Khazaei, M., \& Shafei, M. N. (2020). Cardiovascular Effect of Cuneiform Nucleus } \\
\text { During Hemorrhagic Hypotension. Basic and Clinical Neuroscience, 11(3), 251-260. http://dx.doi.org/10.32598/bcn.11.2.84.4 } \\
\text { dof": } \mathbf{h t t p : / / d x . d o i . o r g / 1 0 . 3 2 5 9 8 / b c n . 1 1 . 2 . 8 4 . 4 ~}\end{array}$ \\
\hline
\end{tabular}

\section{(a) $(0)$}

Article info:

Received: 8 Aug 2018

First Revision: 10 Sep 2018

Accepted: 10 Nov 2019

Available Online: 01 May 2020

Keywords:

Hemorrhage, Cobalt chloride, Cuneiform nucleus, Midbrain Reticular formation, Heart Rate, Blood pressure

\section{A B S T RA C T}

Introduction: The underlying mechanism responsible for the cardiovascular response to Hemorrhage (HEM) is still unknown; however, several brain areas, such as the Cuneiform nucleus $(\mathrm{CnF})$ have shown to be involved. In this study, the cardiovascular effect of the $\mathrm{CnF}$ during HEM was evaluated.

Methods: The animals were divided into the following groups: 1. Vehicle; 2. HEM; 3. Cobalt chloride $\left(\mathrm{CoCl}_{2}\right) ; 4 . \mathrm{CoCl}_{2}+$ saline; and 5. $\mathrm{CoCl}_{2}+\mathrm{HEM}$. Catheterization of the left and right femoral artery was performed to record blood pressure and blood withdrawal, respectively. Saline and $\mathrm{CoCl}_{2}$ were microinjected into the $\mathrm{CnF}$ nucleus, and then blood withdrawal was done for HEM induction. Cardiovascular regulation throughout the experiments was recorded and changes $(\Delta)$ in the Systolic Blood Pressure (SBP), Mean Arterial Pressure (MAP) and Heart Rate (HR) were calculated over time and compared with those treated with saline and HEM, using repeated-measures ANOVA.

Results: HEM significantly reduced $\triangle \mathrm{SBP}$ and $\triangle \mathrm{MAP}$ and augmented $\triangle \mathrm{HR}$ than the vehicle group. $\mathrm{CoCl}_{2}$ did not significantly affect basic $\triangle \mathrm{SBP}, \triangle \mathrm{MAP}$, and $\Delta \mathrm{HR}$ compared with the vehicle group. However, injection of $\mathrm{CoCl}_{2}$ into the $\mathrm{CnF}$ before $\mathrm{HEM}\left(\mathrm{CoCl}_{2}+\mathrm{HEM}\right.$ group $)$ significantly decreased $\triangle \mathrm{SBP}, \triangle \mathrm{MAP}$, and tachycardia, induced by HEM.

Conclusion: Our results indicated that blockade of the $\mathrm{CnF}$ by $\mathrm{CoCl}_{2}$ significantly reduced the hypotension and tachycardia, induced by HEM indicating the involvement of $\mathrm{CnF}$ in cardiovascular regulation during HEM. 


\section{Highlights}

- Inactivation of the $\mathrm{CnF}$ by $\mathrm{CoCl}_{2}$ did not significantly affect basal cardiovascular responses.

- Hemorrhage significantly lowered blood pressure and increased heart rate.

- Inactivation of the $\mathrm{CnF}$ significantly attenuated hypotension and tachycardia induced by hemorrhage.

\section{Plain Language Summary}

The Hemorrhage (HEM) is a life-threatening condition produced by a significant loss of blood, leading to hypotension, decreased tissue perfusion, cellular hypoxia and possibly death. Following HEM, several systems, including the Central Nervous System (CNS) are activated to improve this condition. For example, CNS restores hypotension during HEM and increases tissue perfusion. Numerous brain areas, such as rostral ventrolateral medulla, nucleus tractus solitarius, paraventricular nucleus are involved in the regulation of cardiovascular responses during HEM. Also, other areas, like Cuneiform Nucleus $(\mathrm{CnF})$ are involved in cardiovascular regulation; however, their cardiovascular effects during HEM are unclear. In this study, we evaluated the possible cardiovascular effect of $\mathrm{CnF}$ in HEM. The groups were as follows: vehicle, cobalt chloride $\left(\mathrm{CoCl}_{2}\right.$, a reagent that blocks the activity of nucleus): microinjection of $\mathrm{CoCl}_{2}$ $(1 \mathrm{mM})$ into the CnF; HEM: blood withdrawal of about $15 \%$ of TBV, Hem + saline, and $\mathrm{HEM}+\mathrm{CoCl}_{2}$ groups. In all groups, Blood Pressure (BP) and also Heart Rate (HR) were recorded by a PowerLab instrument and the changes were calculated and compared. The results indicated that HEM decreased BP and increased HR. However, these effects (decreased $\mathrm{BP}$ and increased $\mathrm{HR}$ ) improved only after the inactivation of the $\mathrm{CnF}$. Our results confirmed the involvement of $\mathrm{CnF}$ in cardiovascular regulation of $\mathrm{BP}$ and $\mathrm{HR}$ during HEM.

\section{Introduction}

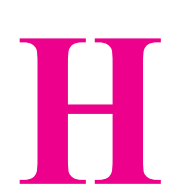

emorrhage (HEM) is a life-threatening incident due to the loss of intravascular volume caused by hypotension with two compensatory and non-compensatory phases (Evans, Ventura, Dampney, \& Ludbrook, 2001; Standl, Annecke, Cascorbi, Heller, Sabashnikov, $\&$ Teske, 2018). The compensatory phase is performed by the elicit of baroreceptors signaling hypotension to the Nucleus of the Solitary Tract (NTS) followed by the activation of vasomotor neurons in Rostral Ventrolateral Medulla (RVLM), an important area in cardiovascular modulation, that excites the sympathetic neurons and maintains blood pressure in normal condition (Naves et al., 2018; Palkovits, 1999). If blood loss continues, the non-compensatory phase, as the next phase, is started, in which sympathetic drive abruptly reduces and arterial pressure falls. The underlying mechanism responsible for these phases of HEM is not completely understood (Evans et al., 2001) and it has suggested that in addition to NTS (Buller, Smith, \& Day, 1999) and RVLM (Dean $\&$ Bago, 2002), other nuclei also are involved. For example, it has reported that the Paraventricular Nucleus of the Hypothalamus (PVN) (Fan \& McIntosh, 1994) and parabrachial areas (Blair \& Mickelsen, 2006) are associated with the cardiovascular regulation during HEM.
Also, numerous nuclei, such as Cuneiform Nucleus $(\mathrm{CnF})$ are found in the brain that play important role in cardiovascular regulation; however, their role in HEM condition has not yet defined (Verberne, 1995).

The $\mathrm{CnF}$ is a reticular nucleus in the midbrain located in the anterolateral of Periaqueductal Grey (PAG) matter and begins from caudate part of the midbrain and continues to the rostral part of pons (Gioia \& Bianchi, 1987). The $\mathrm{CnF}$ is precipitated in several functions, including regulation of motor activity, pain and respiratory modulation, integration of sensory-motor movement related to pain, defense reactions, and sleep (Shafei, Nasimi, Alaei, Pourshanazari, \& Hosseini, 2012; Xiang, Zhu, Guan, \& Ye, 2013). The role of $\mathrm{CnF}$ in cardiovascular regulation has also been reported in previous studies. Verberne reported that electrical stimulation of $\mathrm{CnF}$ increased sympathetic vasomotor outflow and blood pressure (Verberne, 1995). The role of $\mathrm{CnF}$ in cardiovascular regulation has been evaluated (Shafei et al., 2012). For example, our previous study indicated that glutamate increased blood pressure (Nasimi, Shafei, \& Alaei, 2012), and the cholinergic system decreased cardiovascular parameters (Shafei, Niazmand, Hosseini, \& Daloee, 2013). Despite the role of $\mathrm{CnF}$ in cardiovascular regulation, we previously indicated that inactivation of this nucleus by $\mathrm{Co}-$ balt Chloride $\left(\mathrm{CoCl}_{2}\right)$ did not change basic cardiovascu- 
lar parameters (Shafei et al., 2012). Although $\mathrm{CnF}$ is not active in basal conditions, it may affect cardiovascular responses in conditions, such as defense reaction, exercise, pain, and HEM (Verberne, Lam, Owens, \& Sartor, 1997). Similarly, i Ahlgren et al. reported that HEM increased Fos-Like Immunoreactivity (FLI), a marker of brain activity, in the $\mathrm{CnF}$ (Ahlgren, Porter, \& Hayward, 2007). Also, the relationship between $\mathrm{CnF}$ and brain regions, such as RVLM (Verberne, 1995), NTS (Buller, Smith, \& Day, 1999) and PGA mater (Netzer et al., 2011) that regulate cardiovascular response during HEM has been reported. Accordingly, it is conceivable that the $\mathrm{CnF}$ is involved in cardiovascular adjustment during HEM. Therefore, the present study was conducted to evaluate the possible role of $\mathrm{CnF}$ in cardiovascular regulation during HEM.

\section{Metods}

\subsection{Animal and recording of blood pressure}

In this experiment, 30 male Wistar rats were used. Animals anesthetized with urethane $(1.5 \mathrm{~g} / \mathrm{kg}, \mathrm{IP})$. A heating lamp was used to maintain a relatively constant body temperature of the animals. The left femoral artery also was catheterized with an angiocath filled with heparinized saline $(60 \mathrm{u} / \mathrm{ml})$ to record Blood Pressure (BP) and Heart Rate (HR) using a blood pressure transducer and a PowerLab system (ID instrument, Australia) (Shafei \& Nasimi, 2011). In all groups, Systolic Blood Pressure (SBP), Mean Atrial Pressure (MAP), and HR were recorded throughout the trial period.

\subsection{Surgical procedures}

The rats were mounted on a stereotaxic apparatus (Stoelting, USA). The scalp was incised and the skull was leveled between the bregma and lambda, and a small hole drilled above the $\mathrm{CnF}$ in the skull. The $\mathrm{CnF}$ stereotaxic coordinates were -7.6 to $-8.5 \mathrm{~mm}$ caudal to the bregma, -1.7 to $-2.2 \mathrm{~mm}$ lateral to the midline suture, and -5.5 to $-6.2 \mathrm{~mm}$ ventral to the bregma according to the Paxinos and Watson atlas (Paxinos \& Watson 2005). $\mathrm{CoCl}_{2}$ (1mM) (Shafei, Alaei, \& Farrokhi, 2011) was unilaterally microinjected into the $\mathrm{CnF}$ by a single-barreled micropipette (35-45 $\mu \mathrm{m}$ in diameter). For injection, the micropipette was connected through a PE-10 tube to a manual microinjector (Harvard) and carefully introduced into the $\mathrm{CnF}$ and injection was done during $30 \mathrm{~s}$. The volume of the injected drug or saline was 100-150 $\mathrm{nl}$ in all groups (Shafei, Nikyar, Hosseini, Niazmand, \& Paseban, 2017). The study protocol was approved by the Bioethics Committee of Mashhad University of Medical Sciences (Code: 931769).

\subsection{Hemorrhage protocol}

In groups subjected to hemorrhagic hypotension, the right femoral artery was cannulated by a polyethylene tube filled with heparinized saline $(60 \mathrm{u} / \mathrm{ml})$ to prevent clotting during blood withdrawal. Blood withdrawal was initiated 5 min after a microinjection of $\mathrm{CoCl}_{2}$ into the $\mathrm{CnF}$. Blood withdrawal was performed from the femoral artery intermittently at a rate of $1 \mathrm{ml} / 100 \mathrm{~g}$ body weight for 10 min (Dean \& Bago, 2002). In this method, about 15\% of the Total Blood Volume (TBV) was withdrawn. The TBV of the rats was calculated previously as follows: $0.06 \mathrm{ml} /$ $\mathrm{g} \times$ body weight +0.77 . This volume could induce an intermediate hemorrhage suitable to evaluate the role of cardiovascular regulation during HEM (Ahlgren et al., 2007).

At the end of each experiment, the animals' brain was removed and $24 \mathrm{~h}$ after fixation with formalin $10 \%$, serial sections (50-micron thickness) were prepared using a microtome, and the location of the injections verified based on the atlas of Paxinos and a light microscope (Shafei et al., 2012).

\subsection{Drug}

The urethane and $\mathrm{CoCl}_{2}$ were provided by the SigmaCo., USA. All drugs were dissolved in saline.

\subsection{Animal groups}

Animals were randomly divided into the five following groups ( $n=6$ per group): 1 . Vehicle: Microinjection of saline into the $\mathrm{CnF} ; 2$. Cobalt Chloride $\left(\mathrm{CoCl}_{2}\right)$ : Microinjection of $\mathrm{CoCl} 2(1 \mathrm{mM})$ into the $\mathrm{CnF}$; 3. HEM: Blood withdrawal of about $15 \%$ of $\mathrm{TBV}$; 4 . Hem+saline: saline microinjection into the $\mathrm{CnF} 5 \mathrm{~min}$ before $\mathrm{HEM}$; and 5 . $\mathrm{HEM}+\mathrm{CoCl}_{2}$ : $\mathrm{CoCl}_{2}$ microinjection into the $\mathrm{CnF} 5 \mathrm{~min}$ before HEM.

\subsection{Data analysis}

Changes $(\Delta)$ in cardiovascular parameters (SBP, MAP, and $\mathrm{HR}$ ) were calculated and expressed as mean \pm SEM. The $\triangle \mathrm{SBP}, \triangle \mathrm{MAP}$, and $\triangle \mathrm{HR}$ parameters in all groups were computed at each 5 min and statistically compared with the saline and HEM groups (repeated measures ANOVA). Also, the peak $\triangle \mathrm{SBP}, \triangle \mathrm{MAP}$, and $\triangle \mathrm{HR}$ of each group were separately calculated 30 min after HEM (40 min after initiation of HEM) and compared with peak changes of the vehicle and HEM groups (independent sample t-test). A $\mathrm{P}<0.05$ was considered significant. 


\section{Results}

3.1. Effect of the vehicle and $\mathrm{CoCl}_{2}$ microinjection into the $\mathrm{CnF}$ on cardiovascular parameters in normotensive rats

In this study, vehicle (saline) and $\mathrm{CoCl}_{2}$ were separately injected into the $\mathrm{CnF}$. Injection of saline into the $\mathrm{CnF}$ did not significantly change SBP, MAP, and HR compared with pre-injection. Inactivation of the $\mathrm{CnF}$ by microinjection of $\mathrm{CoCl}_{2}$ also did not significantly affect basal cardiovascular parameters than the saline group (Table 1 and Figure 1).

3.2. Effect of hemorrhage on cardiovascular parameters

In HEM, withdrawal of blood was done over $10 \mathrm{~min}$ and cardiovascular responses were evaluated $45 \mathrm{~min}$ later. Figure 2 indicates changes in blood pressure and HR during HEM. Time-course changes in the responses also were plotted in Figures 3-6. As can be seen, at the end of blood withdrawal (10 min after initiation of hemorrhage),
MAP, SBP, and HR decreased and then slowly returned, and after $20 \mathrm{~min}$, SBP and MAP stabilized below the baseline levels; however, HR showed an increase (Figure 2). $\triangle \mathrm{MAP}$ and $\triangle \mathrm{SBP}$ after blood withdrawal were significantly lower compared with the vehicle group over time (repeated measures ANOVA; $\mathrm{P}<0.01, \mathrm{n}=6$ ), whereas HR firstly decreased followed by a significant increase (repeated measures ANOVA; $\mathrm{P}<0.01, \mathrm{n}=6$ ).

Also, $30 \mathrm{~min}$ after blood withdrawal (40 min after initiation of blood withdrawal), the peak $\triangle \mathrm{MAP}, \triangle \mathrm{SBP}$, and $\Delta \mathrm{HR}$ were calculated and compared with the vehicle group. Compared with vehicle group, $\triangle \mathrm{SBP}$ (HEM: $-27.2 \pm 2.8 \mathrm{mmHg}$ vs. vehicle: $-2.3 \pm 1.9 \mathrm{mmHg}, \mathrm{P}<0$. 01 ) and $\triangle$ MAP (HEM: $-21.6 \pm 3.4 \mathrm{mmHg}$ vs. vehicle: $-1.4 \pm 1.6 \mathrm{mmHg}, \mathrm{P}<0.01)$ significantly reduced, whereas $\triangle H R$ (HEM: $64.6 \pm 6.24 \mathrm{mmHg}$ vs. vehicle: $-6.7 \pm 3.8$; $\mathrm{P}<0.001$; independent sample t-test, Figures 3 and 4) significantly increased.
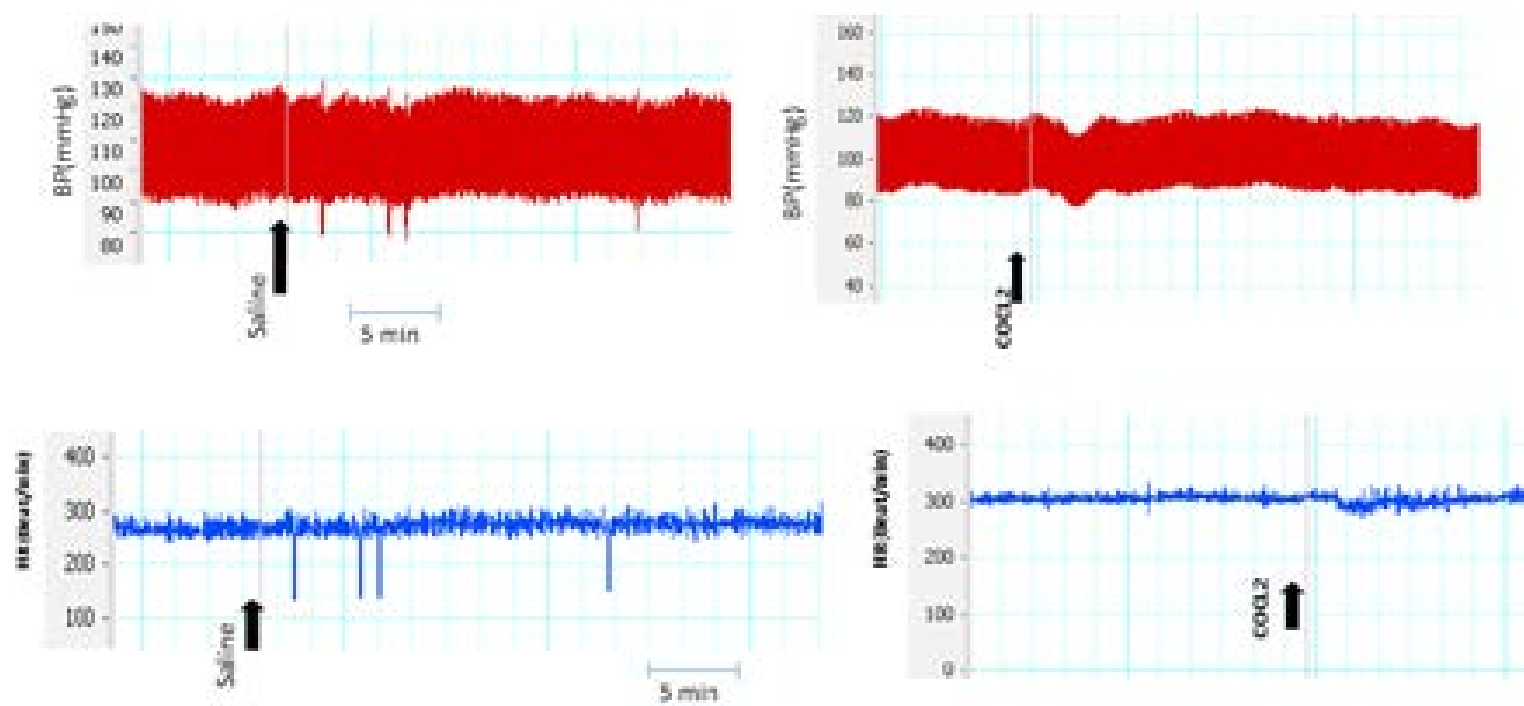

Figure 1. Recording Blood Pressure (BP) and Heart Rate (HR); Arrows indicate injection time.

Table 1. Cardiovascular effect of $\mathrm{CoCl}_{2}$ and saline microinjection into the $\mathrm{CnF}$

\begin{tabular}{cccc}
\hline Group Parameter & SBP $(\mathbf{m m H g})$ & MAP (mmHg) & HR (Beat/min) \\
\hline Vehicle (saline) & $124.2 \pm 2.3$ & $113.5 \pm 1.9$ & $330.6 \pm 7.3$ \\
$\mathrm{CoCl}_{2}$ & $118.5 \pm 1.0$ & $108.8 \pm 1.7$ & $298 \pm 6.9$ \\
\hline
\end{tabular}

Values are presented as Mean \pm SEM and an Independent sample t-test was used for data analysis; $(\mathrm{n}=6)$

SBP: Systolic Blood Pressure; MAP: Mean Arterial Pressure; HR: Heart Rate; CnF: Cuneiform Nucleus; $\mathrm{CoCl}_{2}$ : Cobalt Chloride 

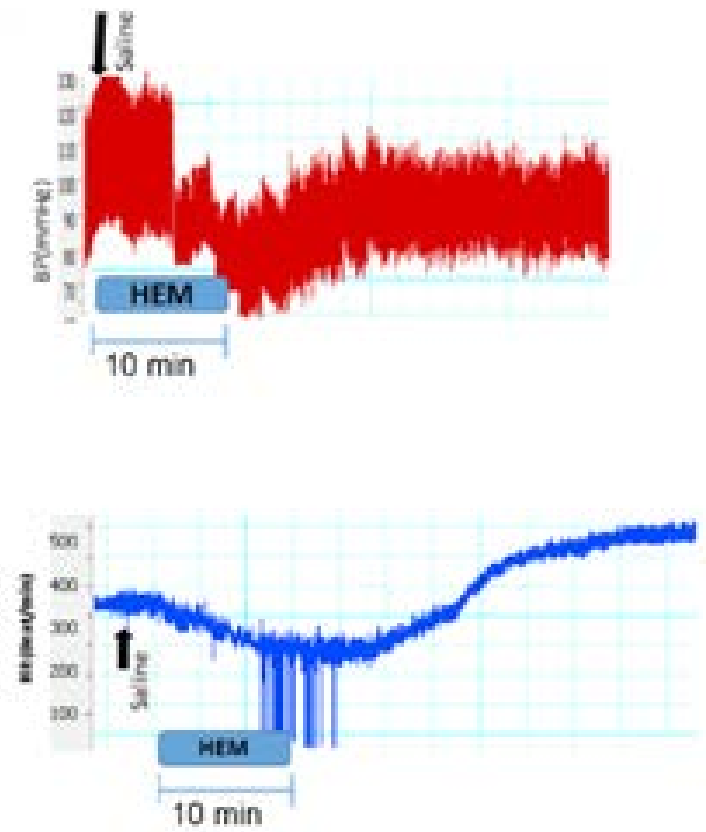
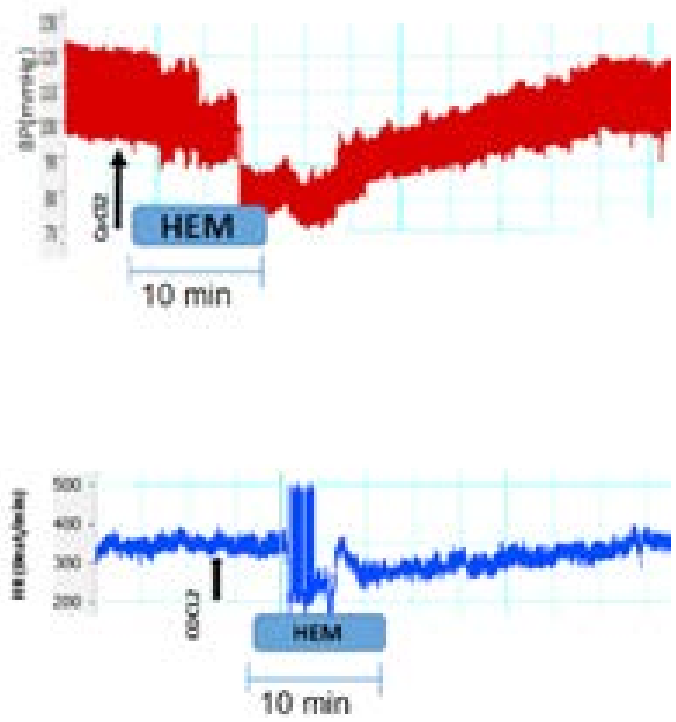

NEUR SSCIENCE

Figure 2. Recording Blood Pressure (BP) and Heart Rate (HR) in the Hemorrhage (HEM) and $\mathrm{HEM}+\mathrm{CoCl}_{2}$ groups; Arrows indicate injection time.

3.3. Inactivation of the $\mathrm{CnF}$ by $\mathrm{CoCl}_{2}$ and its effect on cardiovascular responses in hypotension caused by hemorrhage

In this study, $\mathrm{CnF}$ was first inactivated by the microinjection of $\mathrm{CoCl}_{2}$ and 5 min later, HEM performed over
10 min (Figure 3). Time-course changes of SBP, MAP, and $\mathrm{HR}$ in the HEM, $\mathrm{HEM}+$ saline, and $\mathrm{HEM}+\mathrm{CoCl}_{2}$ groups are shown in Figure 4. As can be seen, in the $\mathrm{HEM}+$ saline group, all parameters were not significant compared with the HEM group; however, they were

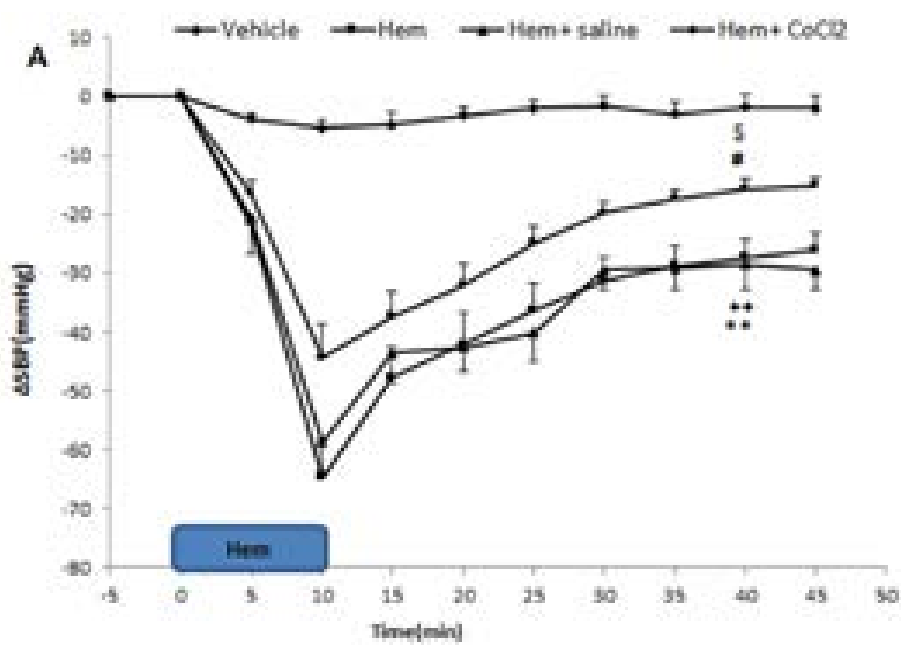

Figure 3. Time courses changes of Systolic Blood Pressure $(\triangle \mathrm{SBP})$ in the experimental groups

HEM significantly decreased SBP than the vehicle group over time $(\mathrm{P}<0.01)$ and this effect was attenuated by cobalt chloride $\left(\mathrm{CoCl}_{2}\right)$, (repeated measures ANOVA, $\left.\mathrm{P}<0.01\right)$. Values are expressed as Mean $\pm S E M ;(n=6)$.

Symbols indicated the peak $\triangle \mathrm{SBP}, 30 \mathrm{~min}$ after blood withdrawal (independent sample t-test)

** $\mathrm{P}<0.001 \mathrm{HEM}$ vs. vehicle group; ${ }^{++} \mathrm{P}<0.01 \mathrm{Hem}+$ saline vs. vehicle group.

${ }^{\#} \mathrm{P}<0.05 \mathrm{HEM}+\mathrm{CoCl}_{2}$ vs. $\mathrm{HEM}$ group; ${ }^{\$} \mathrm{P}<0.05 \mathrm{Hem}+\mathrm{CoCl}_{2}$ vs. Vehicle group 


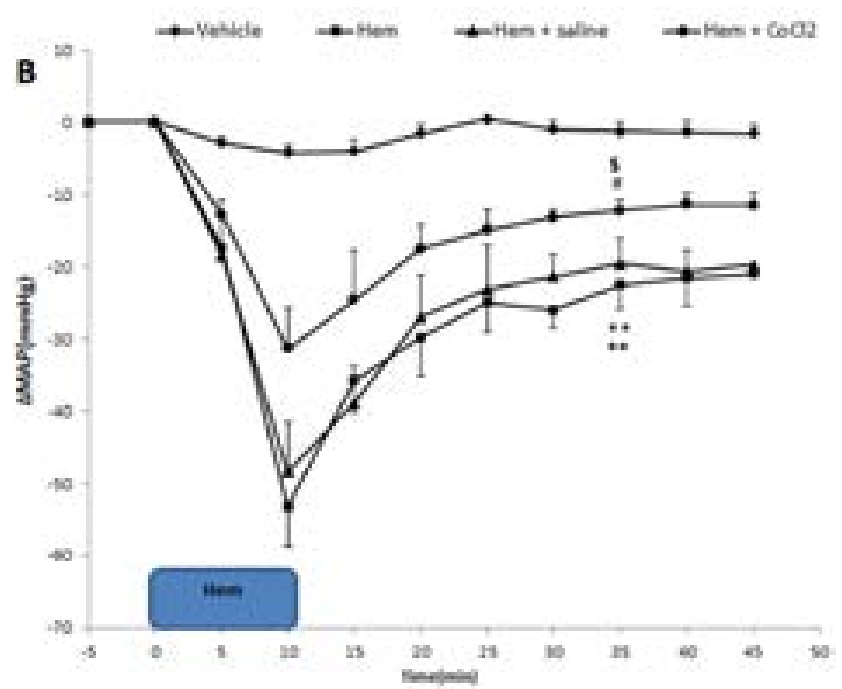

Figure 4. Time courses changes of Mean Arterial Pressure ( $\triangle \mathrm{MAP})$ in the experimental groups

HEM significantly decreased $\triangle \mathrm{MAP}$ than the vehicle group $(\mathrm{P}<0.01)$ and this effect was attenuated by cobalt chloride $\left(\mathrm{CoCl}_{2}\right)$ (repeated measures ANOVA, P<0.01).

Values are expressed as Mean \pm SEM; $(n=6)$.

Symbols indicated the peak $\triangle \mathrm{MAP}, 30 \mathrm{~min}$ after blood withdrawal (Independent sample t-test)

${ }^{* *} \mathrm{P}<0.01 \mathrm{HEM}$ vs. vehicle group; ${ }^{++} \mathrm{P}<0.01 \mathrm{HEM}+$ saline vs. vehicle group

${ }^{\#} \mathrm{P}<0.05 \mathrm{HEM}+\mathrm{CoCl}_{2}$ vs. $\mathrm{HEM}$ group, ${ }^{\$} \mathrm{P}<0.05: \mathrm{HEM}+\mathrm{CoCl}_{2}$ vs. vehicle group

significant than the vehicle group (repeated measures ANOVA, $\mathrm{P}<0.01$, Figure 4). In $\mathrm{HEM}+\mathrm{CoCl}_{2}$ group, both $\triangle \mathrm{MAP}$ and $\triangle \mathrm{SBP}$ significantly increased, whereas $\triangle \mathrm{HR}$ significantly decreased than the HEM group (repeated measures ANOVA, $\mathrm{P}<0$. 01, Figure 4).
The peak $\triangle \mathrm{SBP}, \triangle \mathrm{MAP}$, and $\triangle \mathrm{HR}, 30 \mathrm{~min}$ after blood withdrawal in the $\mathrm{HEM}+$ saline and $\mathrm{HEM}+\mathrm{CoCl}_{2}$ groups were also evaluated. Comparing cardiovascular parameters $30 \mathrm{~min}$ after blood withdrawal $\left(40^{\text {th }} \mathrm{min}\right)$ indicated that in the HEM+saline group, $\triangle \mathrm{SBP}(-28.4 \pm 3.6 \mathrm{mmHg})$ and $\triangle$ MAP $(-20.6 \pm 2.9 \mathrm{mmHg})$ were not significant

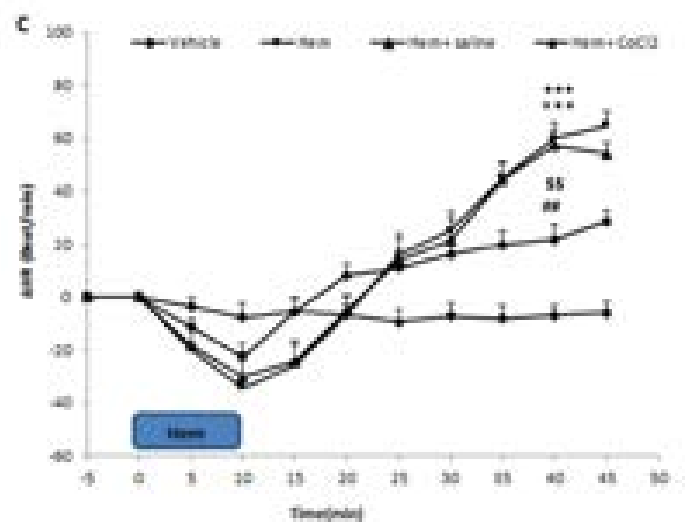

Figure 5. Time courses changes of $\triangle \mathrm{HR}$ during HEM in the experimental groups.

HEM significantly increased than the vehicle group $(\mathrm{P}<0.001)$ and this effect was attenuated by $\mathrm{CoCl}_{2}$ (repeated measures ANOVA, $\mathrm{P}<0.01)$. Values are expressed as Mean \pm SEM; $(\mathrm{n}=6)$.

Symbols indicate the peak $\Delta \mathrm{HR}, 30 \mathrm{~min}$ after blood (independent sample t-test)

${ }^{* * *} \mathrm{P}<0.001 \mathrm{HEM}$ vs. vehicle group; ${ }^{+++} \mathrm{P}<0.001 \mathrm{HEM}+$ saline vs. vehicle group

${ }^{\# \# P}<0.01 \mathrm{HEM}+\mathrm{CoCl}_{2}$ vs. HEM group; ${ }^{\$} \mathrm{P}<0.01 \mathrm{HEM}+\mathrm{CoCl}_{2}$ vs. vehicle group 

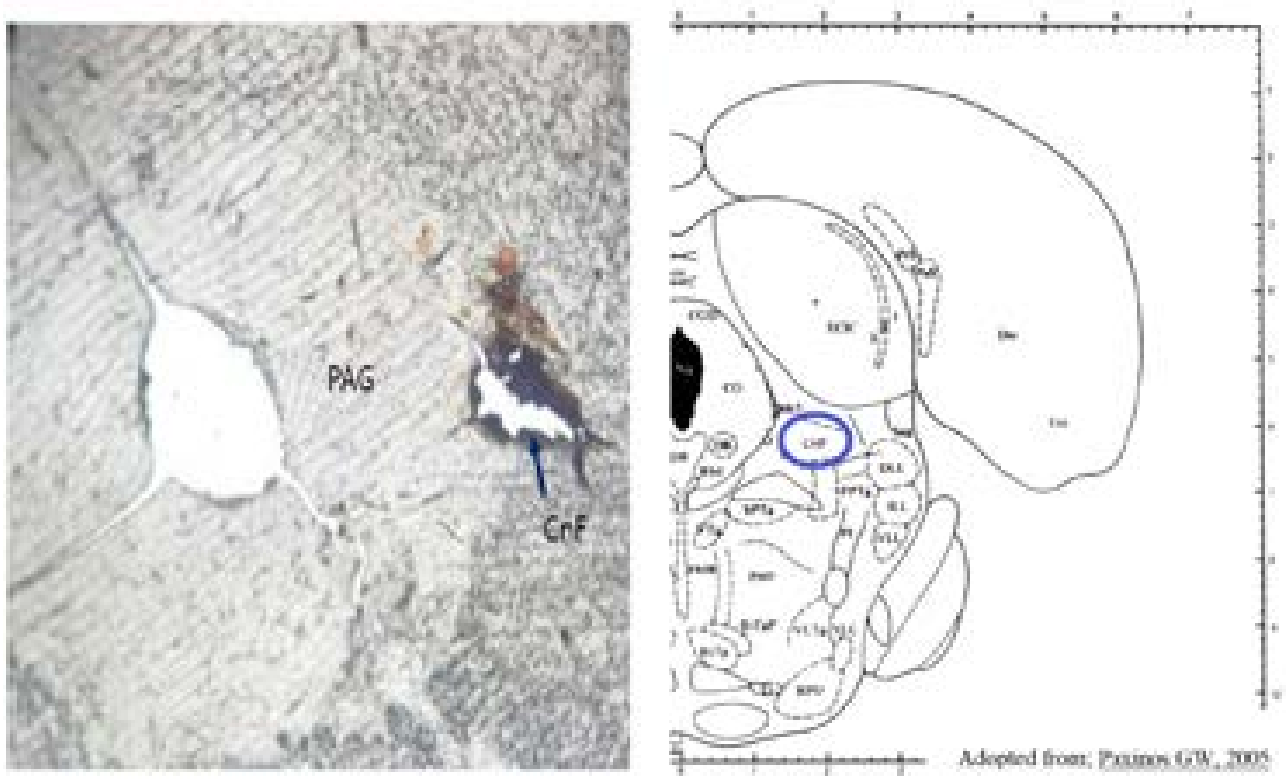

NEUR:SCIENCE

Figure 6. A Photomicrograph of a coronal brain section indicating the microinjection site in the Cuneiform Nucleus $(\mathrm{CnF})$

than the HEM group; however, they were lower than that of the vehicle group $(-2.3 \pm 1.9 \mathrm{mmHg}$ and $-1.4 \pm 1.6$ $\mathrm{mmHg}$, respectively) ( $\mathrm{P}<0.01$, Figure 4$). \Delta \mathrm{HR}$ in the $\mathrm{HEM}+$ saline group was significant in comparison with the vehicle group $(54.3 \pm 5.6$ beats/min vs. $-6.7 \pm 3.8$ beats/ min, respectively; $\mathrm{P}<0.001$, Figure 4).

In the $\mathrm{HEM}+\mathrm{CoCl}_{2}$ group, $30 \mathrm{~min}$ after termination of blood withdrawal, $\triangle \mathrm{SBP}(-15.7 \pm 1.8 \mathrm{mmHg})$ and $\triangle \mathrm{MAP}$ $(12.2 \pm 1.4 \mathrm{mmHg})$ significantly increased than the HEM group $(-27.2 \pm 2.8 \mathrm{mmHg}$ and $-21.6 \pm 3.4 \mathrm{mmHg}$, respectively); ( $\mathrm{P}<0.05$, Figure 4$)$. In the $\mathrm{HEM}+\mathrm{CoCl} 2$ group, $\triangle \mathrm{HR}$ also significantly decreased compared with the HEM group $(28.4 \pm 4.8$ beats/min vs. $64.6 \pm 6.24$ beats/ min, respectively, $\mathrm{P}<0.01$; Figure 4).

\section{Discussion}

We aimed at investigating whether the $\mathrm{CnF}$ is involved in returning the cardiovascular response to the normal level during hemorrhage. Therefore, the role of the $\mathrm{CnF}$ was studied in both normotensive and hemorrhagic hypotensive conditions. Our results indicated that in normotensive condition, inactivation of the $\mathrm{CnF}$ did not affect cardiovascular parameters. This effect confirms our previous experiment, in which $\mathrm{CnF}$ in the basal condition had no significant effect on the cardiovascular system (Shafei et al., 2012). Although CnF nucleus did not cause a cardiovascular response in basal condition; however, inactivation of this nucleus by $\mathrm{CoCl}_{2}$ attenuated hypotension and tachycardia, induced by HEM. This effect confirms the involvement of the $\mathrm{CnF}$ in cardiovascular response during HEM (Shafei et al., 2012). Our results are consistent with those reported by Ahlgren et al. that suggested the involvement of $\mathrm{CnF}$ in cardiovascular regulation during HEM. Algren et al. have shown that blood withdrawal of about $30 \%$ of TBV could initiate a non-compensatory phase and increase FLI in the CnF nucleus (Ahlgren et al., 2007).

Our experiment aimed to maintain the blood pressure of rats in the compensatory phase, i.e. blood withdrawal of $1 \mathrm{ml} / 100 \mathrm{~g} \mathrm{BW}$ in $10 \mathrm{~min}$. Loss of this volume of blood (about 15\%) initially decreased both blood pressure and HR, and then HR significantly increased and blood pressure gradually returned and stabilized at about $30-40 \mathrm{mmHg}$ lower than the baseline value. Reversible inactivation of $\mathrm{CnF}$ with $\mathrm{CoCl}_{2}$ before HEM could attenuate low blood pressure and tachycardia induced by HEM. This effect showed that the $\mathrm{CnF}$ has inhibitory and excitatory effects on blood pressure and HR during HEM, respectively.

The mechanism (s) of this effect of the $\mathrm{CnF}$ is unclear; however, several mechanisms have been proposed. The presence of serotonergic, GABAergic, glutamatergic, cholinergic, and nitrergic neurons have been shown in the CnF (Gioia \& Bianchi, 1987; Shafei \& Nasimi, 2011; Shafei et al., 2013; Shafei et al., 2017; Verberne et al., 1997), of which cholinergic and nitrergic neurons have the inhibitory effect on the cardiovascular system (Shafei et al., 2013; Shafei et al., 2017). We suggest that in HEM, 
these neurons of the $\mathrm{CnF}$ are active and can delay the recovery of blood pressure. However, inactivation of the $\mathrm{CnF}$ nucleus by $\mathrm{CoCl}_{2}$ (a synaptic blocker) blocked Ach release and accelerated the recovery of blood pressure. Other neurons present in the $\mathrm{CnF}$ and those involved in HEM have shown to be serotonergic neurons.

These neurons play an important role in the regulation of cardiovascular responses in normal and HEM conditions. For example, intracerebroventricular injection of serotonin increased both blood pressure and HR or 5-HT1A receptors of the NTS during HEM increased sympathetic activity. Serotonin neurons in $\mathrm{CnF}$ nucleus are associated with several functions $\mathrm{CnF}$ is involved, such as pain regulation. Currently, the role of $\mathrm{CnF}$ neurons in cardiovascular regulation in HEM is unknown and more studies are needed to indicate which neurotransmitter of the $\mathrm{CnF}$ is involved in this function (Gioia \& Bianchi, 1987). Also, the relationship between CnF and RVLM, NTS, PVN, PAG, and Kölliker-Fuse nucleus (KF) areas has previously been reported (Korte, Jaarsma, Luiten, \& Bohus, 1992).

The sympathoexcitatory effect of the $\mathrm{CnF}$ has shown to mostly be mediated by RVLM. However, the direct projection of $\mathrm{CnF}$ to RVLM is spare and indirect and mostly mediated by CnF-KF -RVLM pathway. Our previous study also indicated that the cardiovascular effect of the glutamatergic system of $\mathrm{CnF}$ is mediated via this pathway. As this pathway is excitatory and $\mathrm{CnF}$ has an inhibitory effect in HEM, it can be suggested that this pathway is not important in HEM. Also, CnF is associated with PAG and the cardiovascular effect of $\mathrm{CnF}$ is partly mediated by this Pathway. Due to the involvement of the PAG in the regulation of cardiovascular response during HEM, it is conceivable that this effect of the $\mathrm{CnF}$ can be mediated by the CnF-PAG pathway.

The CnF- raphe pathway can be another possible involved pathway. The relationship between $\mathrm{CnF}$ and raphe nuclei has been shown. Richter et al. indicated that this pathway plays a role in pain modulation (Richter \& Behbehani, 1991). Therefore, it is possible that in addition to pain modulation, it could modulate cardiovascular response during HEM.

In HEM, chemo- and baroreceptors are active and can elicit chemo- and baroreflexes via projection to NTS. A projection from $\mathrm{CnF}$ to NTS has also been documented. Because NTS is an important area in the integration of chemo- and baroreflexes, it is conceivable that $\mathrm{CnF}$ via a relationship with NTS could modulate the activity of chemo- and baroreflexes. Also, Korte et al. indicated the connection of $\mathrm{CnF}$ with NTS and the dorsal motor nucleus of the vagus (Korte et al., 1992). Therefore, is can be suggested that the reduction of tachycardia induced by HEM after blockade of $\mathrm{CnF}$ with $\mathrm{CoCl}_{2}$ is mediated by vagal system or NTS. In this study, $\mathrm{CnF}$ was inactivated unilaterally, which its effect can be different from bilateral inactivation. However, future studies are needed to evaluate the mechanism(s) of the $\mathrm{CnF}$ on cardiovascular regulation during HEM.

In conclusion, our results showed that $\mathrm{CnF}$ nucleus is involved in the adjustment of cardiovascular responses in HEM. Further studies should be conducted to evaluate the mechanisms and neural circuits of $\mathrm{CnF}$ involved in the regulation of cardiovascular response during HEM.

\section{Ethical Considerations}

\section{Compliance with ethical guidelines}

This study was conducted in accordance with the protocols approved by the Ethical Committee of Mashhad University of Medical Sciences (Code: IR.MUMS. REC.1394.200).

\section{Funding}

This study was funded by the Research Council of Mashhad University of Medical Sciences, Mashhad, Iran.

\section{Authors' contributions}

Conceptualization: Mohammad Naser Shafei and Mahmoud Hosseini; Methodology: Reza Mohebbati and Majid Khazaei; Investigation and writing-original draft: Reza Mohebbati; Writing-review \& editing, supervision, and funding acquisition: Mohammad Naser Shafei; Resources: All author.

\section{Conflict of interest}

The authors declared no conflict of interest.

\section{Acknowledgments}

The authors would like to thank the Research Council of Mashhad University of Medical Sciences for funding this study.

\section{References}

Ahlgren, J., Porter, K., \& Hayward, L. F. (2007). Hemodynamic responses and c-Fos changes associated with hypotensive hemorrhage: Standardizing a protocol for severe hemorrhage in 
conscious rats. American Journal of Physiology-Regulatory, Integrative and Comparative Physiology, 292(5), R1862-71. [DOI:10.1152/ ajpregu.00325.2006] [PMID]

Blair, M. L., \& Mickelsen, D. (2006). Activation of lateral parabrachial nucleus neurons restores blood pressure and sympathetic vasomotor drive after hypotensive hemorrhage. American Journal of Physiology-Regulatory, Integrative and Comparative Physiology, 291(3), R742-50. [DOI:10.1152/ajpregu.00049.2006] [PMID]

Buller, K. M., Smith, D. W., \& Day, T. A. (1999). NTS catecholamine cell recruitment by hemorrhage and hypoxia. NeuroReport, 10(18), 3853-6. [DOI:10.1097/00001756-199912160-00024] [PMID]

Dean, C., \& Bago, M. (2002). Renal sympathoinhibition mediated by 5-HT 1A receptors in the RVLM during severe hemorrhage in rats. American Journal of Physiology-Regulatory, Integrative and Comparative Physiology, 282(1), R122-30. [DOI:10.1152/ ajpregu.2002.282.1.R122] [PMID]

Evans, R. G., Ventura, S., Dampney, R. A. L., \& Ludbrook, J. (2001). John Ludbrook APPS symposium neural mechanisms in the cardiovascular responses to acute central hypovolaemia. Clinical and Experimental Pharmacology and Physiology, 28(5-6), 479-87. [DOI:10.1046/j.1440-1681.2001.3473.x] [PMID]

Fan, L., \& McIntosh, T. K. (1994). Effect of dynorphin microinjection in the paraventricular nucleus on the hemodynamic response to hemorrhage in the rat. Circulatory Shock, 42(4), 197-203. [PMID]

Gioia, M., \& Bianchi, R. (1987). The cytoarchitecture of the nucleus cuneiformis. A Nissl and Golgi study. Journal of Anatomy, 155, 16576. [PMID] [PMCID]

Korte, S. M., Jaarsma, D., Luiten, P. G. M., \& Bohus, B. (1992). Mesencephalic cuneiform nucleus and its ascending and descending projections serve stress-related cardiovascular responses in the rat. Journal of the Autonomic Nervous System, 41(1-2), 157-76. [DOI:10.1016/0165-1838(92)90137-6]

Nasimi, A., Shafei, M. N., \& Alaei, H. (2012). Glutamate injection into the cuneiform nucleus in rat, produces correlated single unit activities in the Kolliker-Fuse nucleus and cardiovascular responses. Neuroscience, 223, 439-46. [DOI:10.1016/j.neuroscience.2012.07.041] [PMID]

Naves, L. M., Marques, S. M., Mourão, A. A., Fajemiroye, J. O., Xavier, C. H., \& de Castro, C. H., et al. (2018). Involvement of median preoptic nucleus and medullary noradrenergic neurons in cardiovascular and sympathetic responses of hemorrhagic rats. Scientific Reports, 8(1), 11276. [DOI:10.1038/s41598-018-29310-z] [PMID] [PMCID]

Netzer, F., Bernard, J. F., Verberne, A. J., Hamon, M., Camus, F., \& Benoliel, J. J., et al. (2011). Brain circuits mediating baroreflex bradycardia inhibition in rats: An anatomical and functional link between the cuneiform nucleus and the periaqueductal grey. The Journal of Physiology, 589(Pt 8), 2079-91. [DOI:10.1113/jphysiol.2010.203737] [PMID] [PMCID]

Palkovits, M. (1999). Interconnections between the neuroendocrine hypothalamus and the central autonomic system: Geoffrey Harris Memorial Lecture, Kitakyushu, Japan, October 1998. Frontiers in Neuroendocrinology, 20(4), 270-95. [DOI:10.1006/frne.1999.0186] [PMID]

Paxinos G., \& Watson, C. (2005). The rat brain in stereotaxic coordinates. Burlington, MA: Elsevier Science. https://books.google.com/ books?id=LKJqAAAAMAAJ\&dq
Richter, R. C., \& Behbehani, M. M. (1991). Evidence for glutamic acid as a possible neurotransmitter between the mesencephalic nucleus cuneiformis and the medullary nucleus raphe magnus in the lightly anesthetized rat. Brain Research, 544(2), 279-86. [DOI:10.1016/0006-8993(91)90065-4]

Shafei, M. N., Alaei, H., \& Farrokhi, E. (2011). Effect of reversible inactivation of the Kolliker fuse nucleus on basal blood pressure and heart rate in anesthetized rat. Basic and Clinical Neuroscience, 3(1), 4-8. http:/ / bcn.iums.ac.ir/article-1-189-en.html

Shafei, M. N., \& Nasimi, A. (2011). Effect of glutamate stimulation of the cuneiform nucleus on cardiovascular regulation in anesthetized rats: Role of the pontine Kolliker-Fuse nucleus. Brain Research, 1385, 135-43. [DOI:10.1016/j.brainres.2011.02.046] [PMID]

Shafei, M. N., Nasimi, A., Alaei, H., Pourshanazari, A. A., \& Hosseini, M. (2012). Role of cuneiform nucleus in regulation of sympathetic vasomotor tone in rats. Pathophysiology, 19(3), 151-5. [DOI:10.1016/j.pathophys.2011.11.001] [PMID]

Shafei, M. N., Niazmand, S., Enayatfard, L., Hosseini, M., \& Hasanzade Daloee, M. (2013). Pharmacological study of cholinergic system on cardiovascular regulation in the cuneiform nucleus of rat. Neuroscience Letters, 549, 12-7. [DOI:10.1016/j.neulet.2013.05.046] [PMID]

Shafei, M. N., Nikyar, T., Hosseini, M., Niazmand, S., \& Paseban, M. (2017). Cardiovascular effects of nitrergic system of the pedunculopontine tegmental nucleus in anesthetized rats. Iranian Journal of Basic Medical Sciences, 20(7), 776-82. [DOI:10.22038/ IJBMS.2017.9009] [PMID] [PMCID]

Standl, T., Annecke, T., Cascorbi, I., Heller, A. R., Sabashnikov, A., \& Teske, W. (2018). The nomenclature, definition and distinction of types of shock. Deutsches Ärzteblatt International, 115(45), 757-68. [DOI:10.3238/arztebl.2018.0757] [PMID] [PMCID]

Verberne, A. J. (1995). Cuneiform nucleus stimulation produces activation of medullary sympathoexcitatory neurons in rats. American Journal of Physiology-Regulatory, Integrative and Comparative Physiology, 268(3), R752-8. [DOI:10.1152/ajpregu.1995.268.3.R752] [PMID]

Verberne, A. J., Lam, W., Owens, N. C., \& Sartor, D. (1997). Supramedullary modulation of sympathetic vasomotor function Clinical and Experimental Pharmacology and Physiology, 24(9-10), 74854. [DOI:10.1111/j.1440-1681.1997.tb02126.x] [PMID]

Xiang, H. B., Zhu, W. Z., Guan, X. H., \& Ye, D. W. (2013). The cuneiform nucleus may be involved in the regulation of skeletal muscle tone by motor pathway: A virally mediated trans-synaptic tracing study in surgically sympathectomized mice. Brain, 136(Pt 10) e251. [DOI:10.1093/brain/awt123] [PMID] 
This Page Intentionally Left Blank 